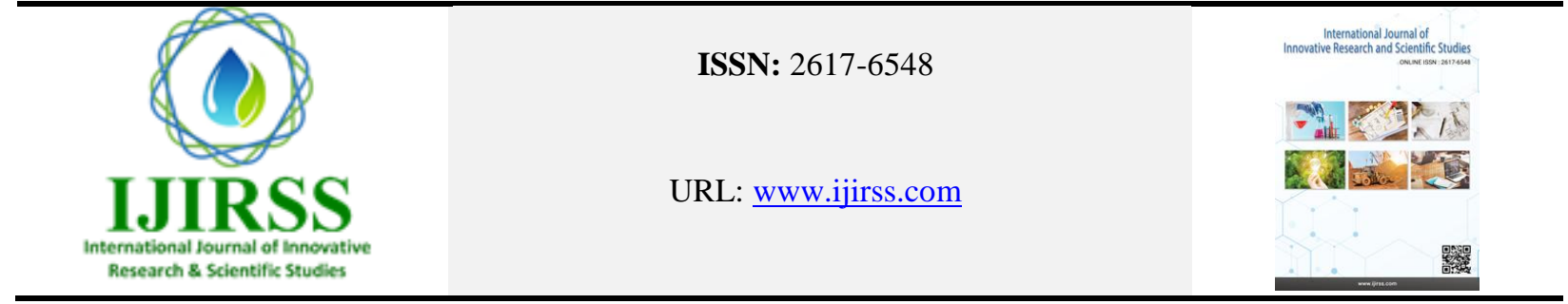

\title{
Prevalence and Antimicrobial Susceptibility Patterns of Staphylococcus aureus/methicillin- resistant Staphylococcus aureus Nasal Carriage among Kabul University Students
}

\author{
Haji Mohammad Naimi ${ }^{1 *}$, Muzhda Haem Rahimi² ${ }^{2}$ Ahmad Zia Noori ${ }^{3}$ \\ ${ }^{1,2,3}$ Department of Microbiology, Faculty of Pharmacy, Kabul University, Kabul, Afghanistan \\ *Corresponding author: Haji Mohammad Naimi (hmnaimi@yahoo.com $)$
}

\begin{abstract}
Nasal carriage of Staphylococcus aureus/Methicillin-resistant Staphylococcus aureus (S. aureus/MRSA) poses a major risk for the transmission and infection of this pathogen especially in hospital setting. The main objective of this study was to investigate the colonization of S. aureus/MRSA among healthy individuals and determine their susceptibility patterns to common antibiotics in Kabul. A total of 150 healthy participants have been included and nasal swabs were collected from all. Samples were cultured on appropriate and selective media for proper identification of S. aureus. Antibiotic susceptibility profiles were determined by the disc diffusion method and interpreted according to the Clinical \& Laboratory Standards Institute (CLSI) protocols. The prevalence of S. aureus nasal colonization was 33.3\% with $12.7 \%$ MRSA nasal carriage. The prevalence of S. aureus/MRSA colonization was not statistically significant according to gender $(\mathrm{p}=0.84)$ and age (0.18). All MRSA isolates were sensitive to rifampicin, linezolid and acid fusidic. Thirty-two percent of the S. aureus isolates were multi-drug resistant. Multi-drug resistance varied from resistance to at least three classes of antibiotics (14\%) to maximum six classes of antibiotics (4\%). This study revealed a high prevalence of S. aureus/MRSA nasal carriage among healthy individuals when compared to similar studies conducted elsewhere and is a reason of concern. Identifying and treating MRSA carriers, as well as responsible use of antibiotics is recommended.
\end{abstract}

Keywords: Staphylococcus aureus, Nasal carriage, MRSA.

DOI: 10.53894 /ijirss.v3i1.25

Funding: Authors thank the World Bank's Higher Education Development Program (HEDP) for financial support.

History: Received: 2 December 2019/Revised: 17 December 2019/Accepted: 31 December 2019/Published: 8 January 2020

Licensed: This work is licensed under a Creative Commons Attribution 4.0 License $(\mathrm{ccc}) \mathrm{Er}$

Acknowledgement: Many thanks as well to Dr. Jeff Armstrong for assisting us in editing the manuscript.

Competing Interests: The authors declare that they have no conflict of interests.

Transparency: The authors confirm that the manuscript is an honest, accurate, and transparent account of the study was reported; that no vital features of the study have been omitted; and that any discrepancies from the study as planned have been explained.

Ethical: This study follows all ethical practices during writing.

\section{Introduction}

Staphylococcus aureus (S. aureus) is a major bacterial human pathogen which is frequently found on the skin and in the upper respiratory tract [1]. It is one of the main bacterial agents responsible for both community and hospital-acquired infections. The acquisition of this pathogen in hospital and community-settings is a serious problem today for public health $[2,3]$. Resistance strains of S. aureus to methicillin and other $\beta$-lactam antibiotics is determined as Methicillin-resistant Staphylococcus aureus (MRSA) and is also is a major problem for public health [4]. MRSA is endemic worldwide in hospitals, and community-associated MRSA (CA-MRSA) is spreading into the community at large [4]. The anterior nares are the principal ecological niche, where the organism colonizes in humans. The nasal carriage of S. aureus increases the 
risk of infection especially in hospital settings [5] and is the major risk factor in the transmission of this pathogen [6]. It has been shown to play a key role in the pathogenesis of S. aureus infections in patients undergoing surgery, dialysis, and intensive care unit (ICU) patients [7]. In a study in a Brazilian university, the nasal carriage for S. aureus was $40.8 \%$ with a rate of 5.8\% MRSA [8]. In this study all isolates of S. aureus were sensitive to linezolid, gentamicin, and cotrimoxazole. Resistance to penicillin G, and ciprofloxacin was found to be $92.0 \%$ and $8.8 \%$, respectively. The prevalence of MRSA among healthy young students in Naresuan University in Thailand were $1 \%$. The researchers found that out of 200 volunteers, $32(16 \%)$ were positive for S. aureus with a 15\% prevalence of the Methicillin-sensitive Staphylococcus aureus (MSSA) [9]. In this study resistance to penicillin, erythromycin, and clindamycin, were $96.7 \%, 26.7 \%$, and $26.7 \%$, respectively. All S. aureus isolates were sensitive to ciprofloxacin, chloramphenicol, gentamicin, cotrimoxazole, rifampicin, linezolid, and fusidic acid. Another study at a dental school in the state of Washington, United States, aimed to determine the prevalence of MRSA nasal carriage in students. This study found that $21 \%$ of these students harbored MRSA [10]. A similar study conducted at the University of Cartagena, Colombia among 387 medical students reported the prevalence of carriage for MSSA and MRSA was $25 \%$ and $1.6 \%$, respectively [11].

The overuse of antibiotics in Afghanistan has led to the increased problem of multi-drugs resistance bacteria such as MRSA. A study conducted at the US military hospital in Bagram Airbase in Afghanistan, showed that patients often carry multidrug-resistant (MDR) bacteria in comparison to US citizens treated at the same hospital [12]. Determination of the prevalence of S. aureus nasal carriage, as well as antimicrobial resistance profiles in healthy population is beneficial for supporting infection control measures. The aim of this study was to assess the prevalence of S. aureus/MRSA, as well as to determine the antimicrobial susceptibility profiles of S. aureus/MRSA isolates to common antibiotics in healthy individuals. The results of this study will be the first information that reports data about MRSA in nasal carriers. It will help medical personnel to understand the prevalence of MRSA in healthy individuals and to take preventative measures against the spread of these strains in the hospital setting.

\section{Materials and Method}

\subsection{Study Population}

This study was conducted in the microbiology laboratory of the faculty of pharmacy of Kabul University over a sixmonth period from February to July, 2019. Samples were collected from anterior nares of 150 volunteer students from different faculties who were admitted in the basic courses, with sterile moistened swab. Informed consent was obtained from all individual participants included in this study. Each swab sample was inoculated on to mannitol salt agar and blood agar base medium (Oxoid, England) to which 5\% sheep blood was added and after 18-24 hours of incubation at $35-370{ }^{\circ} \mathrm{C}$ under aerobic condition, presumptive colonies were isolated. Confirmatory tests were conducted for diagnosis of S. aureus strains by Gram staining, Catalase test, Coagulase test and Pastorex Staph Plus (Bio-Rad, Marnes-la-Coquette, France).

\subsection{Susceptibility Testing}

Confirmed S. aureus strains were subjected to antimicrobial susceptibility testing (AST) by Kirby Bauer disc diffusion method as per Clinical Laboratory Standards Institute (CLSI) guidelines [13] on Muller Hinton agar (Oxoid, England) medium. The following antimicrobial agents were tested: penicillin G, cefoxitin (used for detection of MRSA), erythromycin, cotrimoxazole, gentamicin, tobramycin, clindamycin, chloramphenicol, rifampicin, fosfomycin, kanamycin, acid fusidic, norfloxacin and linezolid. Bacterial suspension was prepared in $5 \mathrm{~mL}$ normal saline solution for AST and the turbidity of the solution was adjusted with 0.5 McFarland standards for obtaining around 1×106 colony forming units (CFU) per mL. Antibiotic discs were placed after $10 \mathrm{~min}$ of inoculation on to Muller Hinton agar seeded with each isolate and were incubated for $18-24 \mathrm{~h}$ at $35-37^{\circ} \mathrm{C}$. The diameter of the zone of inhibition around the disc was measured using sliding metal caliper. For accuracy, during the antibiotic screens, three independent replicates were performed. The susceptibility of all isolates were determined against different classes of antibiotics as follows: In order to detect MRSA we applied the inhibition zone of equal to or more than $24 \mathrm{~mm}$ on Mueller Hinton Agar (MHA) with $30 \mu \mathrm{g}$ cefoxitin disc seeded with growth suspension of S. aureus isolates adjusted to $0.5 \mathrm{McFarland}$ standards at $37{ }^{\circ} \mathrm{C}$ for $18-24 \mathrm{~h}$ [13]. In order to detect Multi-drug resistance, we used the definition of Magiorakos, et al. [14] as non-susceptibility to at least one agent in three or more antimicrobial categories.

\subsection{Statistical Analysis and Quality Assurance}

We used two strains of S. aureus as control. S. aureus ATCC 43300, a mecA positive strain, and S. aureus ATCC 292134, a mecA negative strain; both confirmed with standard PCR using the DNA amplification instrument Mastercycler gradient (Eppendorf, Germany). The statistical analysis was done using SPSS version 20. Binary logistic regression was used to determine the association between MRSA infection, age and gender. Chi-square test was used to compare susceptibility to common antimicrobial agents between MSSA and MRSA isolates. A P-value less than 0.05 was considered statistically significant.

\section{Results}

Of the 150 healthy students from Kabul university, 50 (33.3\%) were positive for S. aureus. About $19(38 \%)$ isolates were identified as MRSA. The prevalence of MRSA nasal carriage was $12.7 \%$. Of 50 nasal carriers, $36(72.0 \%)$ were male and $14(28.0 \%)$ were female. Their mean age was 22 years (19-38 y, median $22 \mathrm{y})$. The difference of MRSA isolation was not statistically significant according to gender $(\mathrm{p}=0.84)$ and age $(\mathrm{p}=0.18)$. All strains $(50 ; 100 \%)$ were susceptible to rifampicin and linezolid. Isolates displayed a low resistance rate to erythromycin (32\%), clindamycin (8\%), norfloxacin 
(18\%), gentamicin (6\%), kanamycin (30\%), tobramycin (22\%), cotrimoxazole (18\%), acid fusidic (4\%), chloramphenicol $(2 \%)$, and fosfomycin ( $8 \%)$. The majority $(96 \%)$ of S. aureus isolates were resistant to penicillin (Table 1).

Table-1.

Antimicrobial susceptibility patterns of S. aureus isolates to different antimicrobial agents from nasal carriers.

\begin{tabular}{l|l|l|l}
\hline Classes of ATB & Antibiotics & Sensitive N (\%) & Resistant N (\%) \\
\hline Penicillines & Penicillin G & $2(4.0)$ & $48(96.0)$ \\
\hline Macrolides & Erythromycin & $34(68.0)$ & $16(32.0)$ \\
\hline Quinolones & Norfloxacin & $41(82.0)$ & $9(18.0)$ \\
\hline Sulfonamides & Cotrimoxazole & $41(82.0)$ & $9(18.0)$ \\
\hline \multirow{2}{*}{ Aminoglycosides } & Gentamicin & $47(94.0)$ & $3(6.0)$ \\
\cline { 2 - 4 } & Tobramycin & $39(78.0)$ & $11(22.0)$ \\
\hline Lincosamides & Kanamycin & $35(70.0)$ & $15(30.0)$ \\
\hline Oxazolidinone & Clindamycin & $46(92.0)$ & $4(8.0)$ \\
\hline Divers & Linezolid & $50(100.0)$ & $0(0.0)$ \\
\hline & Rifampicin & $50(100.0)$ & $0(0.0)$ \\
\cline { 2 - 4 } & Chloramphenicol & $49(98.0)$ & $1(2.0)$ \\
\hline & Acid fusidic & $48(96.0)$ & $2(4.0)$ \\
\hline & Fosfomycin & $46(92.0)$ & $4(8.0)$ \\
\hline
\end{tabular}

The resistance rates of MRSA isolates to common antimicrobials were not statistically significant to those of MSSA isolates. We did not find any strain of MSSA to be resistant to chloramphenicol, rifampicin and linezolid. 96.8\% of MSSA were susceptible to gentamicin and $93.5 \%$ to clindamycin, $93.5 \%$ to acid fusidic and $90.3 \%$ to fosfomycin, while $100 \%$ of MRSA strains were susceptible to linezolid, rifampicin and acid fusidic. Susceptibility to erythromycin was low in both MSSA (71.0\%) and MRSA (63.2\%). MSSA vs MRSA isolates showed a higher susceptibility to norfloxacin, gentamicin, clindamycin and chloramphenicol.

Table-2.

Comparative susceptibility of MRSA and MSSA strains to different antimicrobial agents.

\begin{tabular}{l|l|l|l|l}
\hline Classes of ATB & Antibiotics & MSSA (\%) & MRSA (\%) & P-value \\
\hline Penicillines & Penicillin G & 6.5 & 0.0 & 0.519 \\
\hline Macrolides & Erythromycin & 71.0 & 63.2 & 0.756 \\
\hline Quinolones & Norfloxacin & 87.1 & 73.7 & 0.273 \\
\hline Sulfonamides & Cotrimoxazole & 80.6 & 84.2 & 0.999 \\
\hline \multirow{2}{*}{ Aminoglycosides } & Gentamicin & 96.8 & 89.5 & 0.549 \\
\cline { 2 - 5 } & Tobramycin & 71.0 & 89.5 & 0.170 \\
\hline \multirow{2}{*}{ Lincosamides } & Kanamycin & 67.7 & 73.7 & 0.757 \\
\hline Oxazolidinone & Clindamycin & 93.5 & 89.5 & 0.629 \\
\hline \multirow{2}{*}{ Divers } & Linezolid & 100 & 100 & - \\
\hline & Rifampicin & 100 & 100 & - \\
\cline { 2 - 5 } & Chloramphenicol & 100 & 94.7 & 0.380 \\
\hline & Acid fusidic & 93.5 & 100 & 0.519 \\
\hline & Fosfomycin & 90.3 & 94.7 & 0.999 \\
\hline
\end{tabular}

All MRSA isolates were sensitive to linezolid, rifampicin and acid fusidic. For further information, please refer to Table 2.

\subsection{Multi-Drug Resistance (MDR) Profile of S. Aureus}

Sixteen $(32 \%)$ of the isolates were multi-drug resistant. Multi-drug resistant strains ranged from resistance to three classes of antibiotics (14\%) to six classes of antibiotics (4\%). Details of resistance to different antibiotics are described in Table 3.

\section{Discussion}

There is no previous study regarding prevalence of $\mathrm{S}$. aureus and particularly MRSA nasal carriage in healthy individuals in Afghanistan until now. In this study we found the percentage of nasal carrier for S. aureus to be $33.3 \%$ with a prevalence of 38\% MRSA. In a similar study in Pakistan which is neighbor of Afghanistan, the nasal carriage for S. aureus was $18.2 \%$ with a prevalence of $1.5 \%$ MRSA [15]. Other studies demonstrate that the prevalence of nasal MRSA colonization was $1.5 \%$ in UK [16] $0.1 \%$ in Poland [17] 3.6\% in Ethiopia [18] 2\% in Malaysia [19] and 3\% in China [20]. Most of the studies demonstrate that the MRSA carriage rate was lower than in our study in Afghanistan. In this study, the frequency of MRSA nasal carriage was higher in male than female, but not statistically significant between male and female students $(\mathrm{p}=0.84)$, which shows that gender is not a risk factor for MRSA nasal colonization. This in accordance to our previous study among clinical samples of S. aureus in Kabul health facilities, which also showed that there is no 
association between gender and MRSA infection [21]. This is also in agreement with earlier reports by Geyid and Lemeneh [22] indicating that gender and age are not risk factors for the acquisition or colonization of MRSA.

Table-3.

Percentage of resistance patterns of S. aureus isolates to different number of antibiotics.

\begin{tabular}{l|l|l}
\hline \multirow{2}{*}{ Antibiotic } & Resistant strains & \\
\cline { 2 - 3 } & No of S. aureus & \% \\
\hline No resistance to antibiotics & 1 & 2.0 \\
\hline COT & 1 & 48.0 \\
P & 23 & \\
\hline P, ERY & 2 & 10.0 \\
P, FOS & 2 & \\
P, NOR & 1 & 14.0 \\
\hline P, ERY, AF & 1 & \\
P, TOB, KAN & 3 & \\
P, KAN, ERY & 1 & \\
P, COT, AF & 1 & 10.0 \\
P, ERY, CLI & 1 & \\
\hline P, ERY, NOR, COT & 1 & \\
P, KAN, TOB, NOR & 1 & \\
P, KAN, TOB, GEN & 1 & \\
P, KAN, TOB, ERY & 1 & 6.0 \\
P, ERY, CLI, COT & 1 & \\
\hline P, KAN, TOB, GEN, ERY & 1 & \\
P, KAN, ERY, NOR, COT & 1 & \\
P, KAN, TOB, ERY, COT & 1 & \\
\hline P, KAN, ERY, CLI, NOR, CHL & 1 & \\
P, KAN, ERY, NOR, COT, FOS & 1 & \\
P, KAN, TOB, ERY, NOR, COT & 1 & \\
\hline P, KAN, TOB, GEN, ERY, NOR, FOS & 1 & \\
P, KAN, TOB, ERY, CLI, NOR, COT & 1 & \\
\hline Total & 50 & \\
\hline COT: cotrimoxazole, P: penicillin, ERY: erythromycin, FOS: fosfomycin, NOR: norfloxacin, AF: acid \\
fusidic, TOB: tobramycin, KAN: kanamycin, GEN: gentamicin, CLI: clindamycin, CHL: chloramphenicol. \\
& & \\
\hline
\end{tabular}

The main limitation of this study is that only MRSA isolates collected from nasal carriers among Kabul university students were included. Therefore, the results do not reflect the global epidemiology in Afghanistan and are only representative of MRSA epidemiology of nasal carriage among a limited number of university students. In this study, despite the high prevalence of MRSA, there was no isolate resistant to rifampicin, linezolid and acid fusidic, while in our previous study about prevalence of MRSA in clinical samples $8.5 \%$ were resistant to rifampicin [21].

The study showed that these antibiotics are potent antimicrobial agents for decolonization of nasal MRSA carriers in healthy individuals. Interestingly most of MRSA isolates were susceptible to gentamicin and tobramycin (89.5\%), kanamycin $(73.7 \%)$, clindamycin $(89.5 \%)$, chloramphenicol and fosfomycin $(94.7 \%)$, cotrimoxazole $(84.2 \%)$, norfloxacin (73.2\%) and erythromycin $(63.2 \%)$, which showed they are more sensitive to common antibiotics compared to our previous study about clinical MRSA isolates in Kabul [21]. Interestingly the susceptibility rate of MRSA isolates to fosfomycin, acid fusidic, tobramycin, kanamycin, and cotrimoxazole was higher than MSSA isolates but not statistically significant. The prevalence of multi-drug resistance was $32 \%$ in this study which is considerably high compared to a similar study in Saudi Arabia which showed to be $8 \%$ [23] however this is very low compared to clinical isolates of MRSA in Kabul which showed to be $91.4 \%$ [21].

\section{Conclusion}

The prevalence of S. aureus/MRSA nasal carriage among healthy individuals in this study was relatively high when compared to similar studies conducted elsewhere and is a matter of concern. Susceptibility patterns of the isolates demonstrated that multidrug resistance is emerging among community strains of $\mathrm{S}$. aureus, and individuals who are harboring these isolates can act as reservoirs and a risk factor for transmission and outbreak of MRSA infection. Fortunately, no MRSA isolate was resistant to linezolid, rifampicin and acid fusidic and they can be used as potent antimicrobial agents for MRSA nasal decolonization. Identifying and treating MRSA carriers, as well as responsible use of antibiotics is recommended. We recommend more similar studies on healthy individuals in other provinces of Afghanistan, focusing on prevalence of MRSA nasal carriage.

\section{References}

[1] F. D. Lowy, "Staphylococcus aureus infections," New England Journal of Medicine, vol. 339, pp. 520-532, 1998. 
[2] R. Williams, "Healthy carriage of Staphylococcus aureus: Its prevalence and importance," Bacteriological Reviews, vol. 27, pp. 56-71, 1963. Available at: https://doi.org/10.1128/mmbr.27.1.56-71.1963.

[3] A. Gnanamani, P. Hariharan, and M. Paul-Satyaseela, "Staphylococcus aureus: Overview of bacteriology, clinical diseases, epidemiology, antibiotic resistance and therapeutic approach," Frontiers in Staphylococcus Aureus, vol. 4, p. $28,2017$. Available at: https://doi.org/10.5772/67338.

[4] M. Goetghebeur, P.-A. Landry, D. Han, and C. Vicente, "Methicillin-resistant Staphylococcus aureus: A public health issue with economic consequences," Canadian Journal of Infectious Diseases and Medical Microbiology, vol. 18, pp. 27-34, 2007.

[5] J. Kluytmans and H. Wertheim, "Nasal carriage of Staphylococcus aureus and prevention of nosocomial infections," Infection, vol. 33, pp. 3-8, 2005. Available at: https://doi.org/10.1007/s15010-005-4012-9.

[6] H. F. Wertheim, D. C. Melles, M. C. Vos, W. van Leeuwen, A. van Belkum, H. A. Verbrugh, and J. L. Nouwen, "The role of nasal carriage in Staphylococcus aureus infections," The Lancet Infectious Diseases, vol. 5, pp. 751-762, 2005.

[7] A. Sakr, F. Brégeon, J.-L. Mège, J.-M. Rolain, and O. Blin, "Staphylococcus aureus nasal colonization: An update on mechanisms, epidemiology, risk factors, and subsequent infections," Frontiers in Microbiology, vol. 9, p. $2419,2018$. Available at: https://doi.org/10.3389/fmicb.2018.02419.

[8] K. A. Prates, A. M. Torres, L. B. Garcia, S. F. Y. Ogatta, C. L. Cardoso, and M. C. B. Tognim, "Nasal carriage of methicillinresistant Staphylococcus aureus in university students," Brazilian Journal of Infectious Diseases, vol. 14, pp. 316-318, 2010.

[9] T. Kitti, K. Boonyonying, and S. Sitthisak, "Prevalence of methicillin-resistant Staphylococcus aureus among university students in Thailand," Southeast Asian Journal of Tropical Medicineand Public Health, vol. 42, p. 1498, 2011.

[10] M. C. Roberts, O. O. Soge, J. A. Horst, K. A. Ly, and P. Milgrom, "Methicillin-resistant Staphylococcus aureus from dental school clinic surfaces and students," American Journal of Infection Control, vol. 39, pp. 628-632, 2011. Available at: https://doi.org/10.1016/j.ajic.2010.11.007.

[11] A. Bettin, C. Causil, and N. Reyes, "Molecular identification and antimicrobial susceptibility of Staphylococcus aureus nasal isolates from medical students in Cartagena, Colombia," Brazilian Journal of Infectious Diseases, vol. 16, pp. 329-334, 2012. Available at: https://doi.org/10.1016/j.bjid.2012.06.017.

[12] D. E. Sutter, L. U. Bradshaw, L. H. Simkins, A. M. Summers, M. Atha, R. L. Elwood, J. L. Robertson, C. K. Murray, G. W. Wortmann, and D. R. Hospenthal, "High incidence of multidrug-resistant gram-negative bacteria recovered from Afghan patients at a deployed US military hospital," Infection Control \& Hospital Epidemiology, vol. 32, pp. 854-860, 2011. Available at: https://doi.org/10.1086/661284.

[13] N. C. f. C. L. Standards and A. L. Barry, Methods for determining bactericidal activity of antimicrobial agents: Approved guideline vol. 19. Wayne, PA: National Committee for Clinical Laboratory Standards, 1999.

[14] A.-P. Magiorakos, A. Srinivasan, R. Carey, Y. Carmeli, M. Falagas, C. Giske, S. Harbarth, J. Hindler, G. Kahlmeter, and B. Olsson-Liljequist, "Multidrug-resistant, extensively drug-resistant and pandrug-resistant bacteria: An international expert proposal for interim standard definitions for acquired resistance," Clinical Microbiology and Infection, vol. 18, pp. 268-281, 2012. Available at: https://doi.org/10.1111/j.1469-0691.2011.03570.x.

[15] C. A. Boncompain, C. A. Suárez, and H. R. Morbidoni, "Staphylococcus aureus nasal carriage in health care workers: First report from a major public hospital in Argentina," Argentine Journal of Microbiology, vol. 49, pp. 125-131, 2017. Available at: https://doi.org/10.1016/j.ram.2016.12.007.

[16] L. Abudu, I. Blair, A. Fraise, and K. Cheng, "Methicillin-resistant Staphylococcus aureus (MRSA): A community-based prevalence survey," Epidemiology \& Infection, vol. 126, pp. 351-356, 2001.

[17] K. Szymanek-Majchrzak, J. Kosiński, K. Żak, K. Sułek, A. Młynarczyk, and G. Młynarczyk, "Prevalence of methicillin resistant and mupirocin-resistant Staphylococcus aureus strains among medical students of Medical University in Warsaw," Przegl Epidemiol, vol. 73, pp. 39-48, 2019. Available at: https://doi.org/10.32394/pe.73.05.

[18] F. Efa, Y. Alemu, G. Beyene, E. K. Gudina, and W. Kebede, "Methicillin-resistant Staphylococcus aureus carriage among medical students of Jimma University, Southwest Ethiopia," Heliyon, vol. 5, pp. 11-19, 2019.

[19] Z. Suhaili, P. A. Rafee, N. M. Azis, C. C. Yeo, S. A. Nordin, A. R. Abdul Rahim, and M. M. Jamil Al-Obaidi, "Characterization of resistance to selected antibiotics and Panton-Valentine leukocidin-positive Staphylococcus aureus in a healthy student population at a Malaysian University," Germs, vol. 8, pp. 21-30, 2018.

[20] J. Du, C. Chen, B. Ding, J. Tu, Z. Qin, C. Parsons, C. Salgado, Q. Cai, Y. Song, and Q. Bao, "Molecular characterization and antimicrobial susceptibility of nasal Staphylococcus aureus isolates from a Chinese medical college campus," PLoS One, vol. 6, p. e27328, 2011. Available at: https://doi.org/10.1371/journal.pone.0027328.

[21] H. M. Naimi, H. Rasekh, A. Z. Noori, and M. A. Bahaduri, "Determination of antimicrobial susceptibility patterns in Staphylococcus aureus strains recovered from patients at two main health facilities in Kabul, Afghanistan," BMC Infectious Diseases, vol. 17, pp. 1-7, 2017. Available at: https://doi.org/10.1186/s12879-017-2844-4.

[22] A. Geyid and Y. Lemeneh, "The incidence of methicillin resistant S. aureus strains in clinical specimens in relation to their beta-lactamase producing and multiple-drug resistance properties in Addis Abeba," Ethiopian Medical Journal, vol. 29, pp. 149-161, 1991.

[23] A. Alghaithy, N. Bilal, M. Gedebou, and A. Weily, "Nasal carriage and antibiotic resistance of Staphylococcus aureus isolates from hospital and non-hospital personnel in Abha, Saudi Arabia," Transactions of the Royal Society of Tropical Medicine and Hygiene, vol. 94, pp. 504-507, 2000. Available at: https://doi.org/10.1016/s0035-9203(00)90066-x. 